TITLE:

\title{
Investigation of high-Q channel drop filters using donor-type defects in two-dimensional photonic crystal slabs
}

AUTHOR(S):

Akahane, Y; Asano, T; Song, BS; Noda, S

\section{CITATION:}

Akahane, Y ... [et al]. Investigation of high-Q channel drop filters using donor-type defects in two-dimensional photonic crystal slabs. APPLIED PHYSICS LETTERS 2003, 83(8): 15121514

ISSUE DATE:

2003-08-25

URL:

http://hdl.handle.net/2433/50218

\section{RIGHT:}

Copyright 2003 American Institute of Physics. This article may be downloaded for personal use only. Any other use requires prior permission of the author and the American Institute of Physics. 


\title{
Investigation of high- $Q$ channel drop filters using donor-type defects in two-dimensional photonic crystal slabs
}

\author{
Yoshihiro Akahane, ${ }^{\text {a) }}$ Takashi Asano, Bong-Shik Song, and Susumu Nodab) \\ Department of Electronic Science and Engineering, Kyoto University, Core Research for Evolutional \\ Science and Technology (CREST), Japan Science and Technology Corporation (JST), Kyoto 606-8501, Japan
}

(Received 25 February 2003; accepted 25 June 2003)

\begin{abstract}
This letter describes experimental investigations of surface-emitting channel drop filters using donor-type point defect cavities and line-defect waveguides in two-dimensional photonic crystal slabs. By using donor-type defect cavities with three and four linearly aligned missing air holes, filter quality factors of around 2600 and 6400, respectively, are achieved experimentally, compared to the quality factor of 400 of previous acceptor-type defect cavities. Radiation patterns and polarization properties of light emitted from the defects are also discussed. The results indicate that these donor-type defects are very useful for the development of ultrasmall high-performance channel add/drop filters. (C) 2003 American Institute of Physics. [DOI: 10.1063/1.1604179]
\end{abstract}

A two-dimensional (2D) photonic crystal (PC) slab ${ }^{1-9}$ has attracted much attention as a relatively easy material in which to achieve a gap in the photonic mode spectrum. We have previously reported a very interesting phenomenon in which photons propagating along a line defect (waveguide) are trapped and emitted to free space by a single-point defect created in a 2D PC slab when the photon frequency matches the defect frequency [see Fig. 1(a)]. ${ }^{1}$ This phenomenon can be applied to ultrasmall surface-emitting channel add/drop filters for wavelength division multiplexed (WDM) optical communication systems.

Up until now, our experiments have mainly focused on devices which utilize acceptor-type defects (enlarged airhole rods) in 2D PC slabs with a triangular lattice of air holes. ${ }^{1,3,4}$ Although various device performance properties, such as wide tunability of dropped wavelength and high drop efficiency have been demonstrated, the quality $(Q)$ factors of these devices, which determine the resolution of filtering operation, are at most 400 . For practical applications such as dense WDM (D-WDM) systems, the $Q$ factors of these devices need be increased much further. In our last work, ${ }^{7}$ we theoretically investigated various kinds of donor-type defects apart from acceptor-type defects in an attempt to realize high- $Q$ cavities, paying close attention to other characteristics required in channel add/drop filters (such as spatial patterns and polarizations of the vertically emitted light, and free spectral range). From these theoretical investigations, a donor-type defect, composed of three linearly aligned missing air holes, was found to be very promising. ${ }^{7}$

In this work, a channel add/drop device is fabricated which utilizes a donor-type defect composed of three linearly aligned missing air holes (defined as L3), and the important properties of this device, such as $Q$ factors and radiation patterns, are investigated experimentally. A device utilizing a defect composed of four linearly aligned missing air holes

\footnotetext{
a) On leave from Advanced Materials R\&D Laboratories, Sumitomo Electric Industries, Ltd., Itami, Hyogo 664-0016, Japan; electronic mail: y-akahane@sei.co.jp

b)Electronic mail: snoda@kuee.kyoto-u.ac.jp
}

(defined as L4) is also investigated, with an expectation of further improvements in $Q$ factor.

The sample investigated was an air-bridge triangular lattice PC slab fabricated from $0.25-\mu \mathrm{m}$-thick Si. The lattice constant $(a)$ of the PC and radii of the air holes were 0.42 $\mu \mathrm{m}$ and $0.12 \mu \mathrm{m}$, respectively. In this structure, the photonic band gap (PBG) is open only for transverse-electric (TE) mode photons in a frequency range of $0.256-0.320(c / a)$, where $c$ is the velocity of light in a vacuum. (Since the PBG is not open for transverse-magnetic mode photons, defect properties were investigated only about TE-like polarization in this work.) A line-defect waveguide and donor-type defect (L3 or L4) were introduced by not making air holes in a row along the same $\Gamma-J$ direction. The distance between the line and point defects $(d)$ [see Fig. 1(a)] was chosen so as to maximize drop efficiency. Based on a previous theoretical

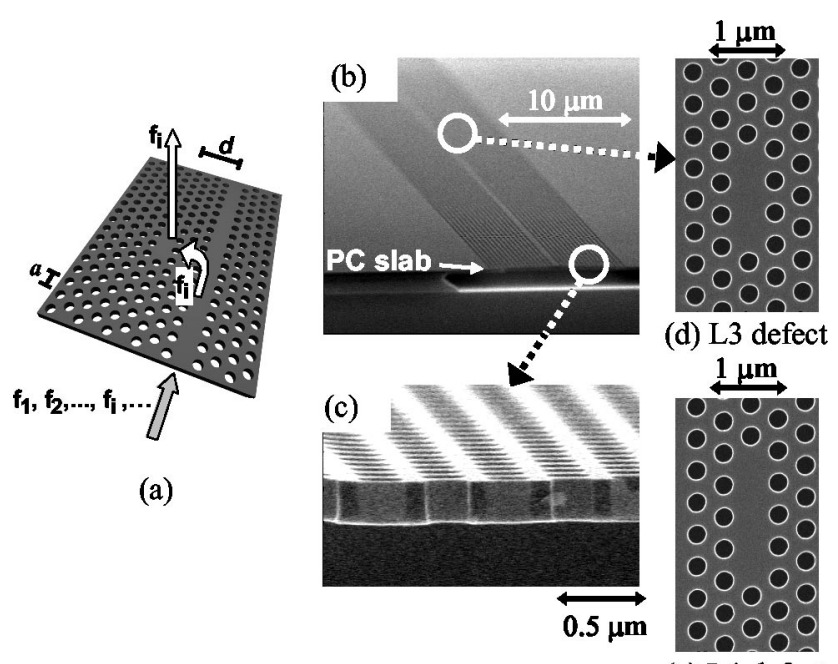

(e) LA defect

FIG. 1. (a) Schematic of a surface-emitting channel add/drop filtering device consisting of a 2D PC slab with a line-defect waveguide and a single point defect. The definitions of lattice constant $a$ and distance between the waveguide and point defect $d$ are also shown. (b)-(e) Scanning electron microscope images of the fabricated samples; (b) overall view, (c) magnified view near the facet, (d) top view of L3 (three linearly aligned missing air holes) defect, and (e) top view of L4 (four linearly aligned missing air holes) defect. 


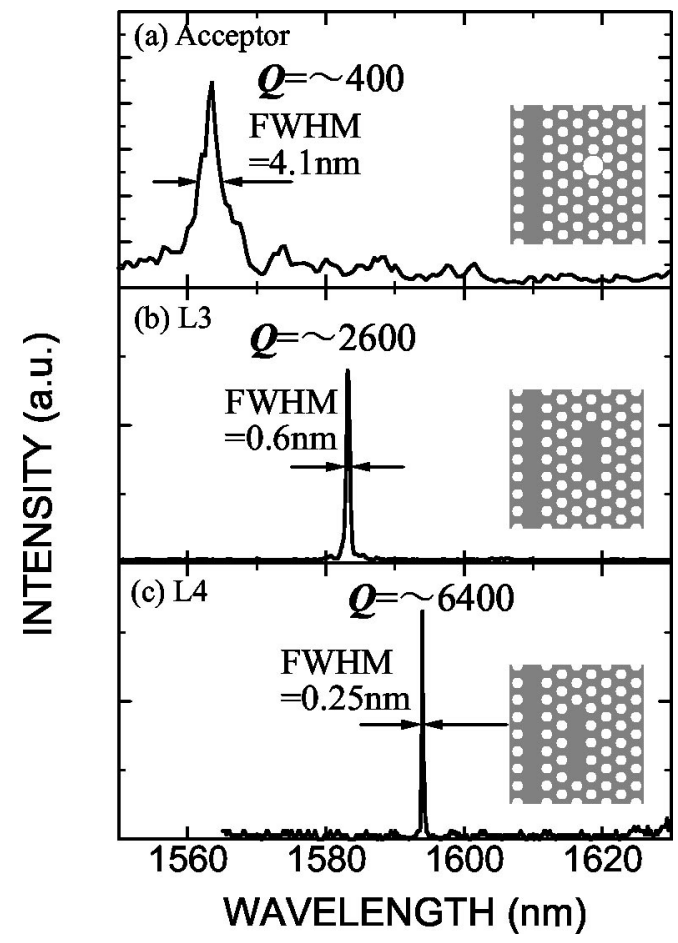

FIG. 2. Channel drop spectra of samples composed of a waveguide and (a) acceptor-type, (b) L3 donor-type, and (c) L4 donor-type point defects. The distances between the point defect and waveguide in (a), (b), and (c) are four, four, and three rows of air holes, respectively. $Q$ factors of the L3 and L4 donor-type defects are much higher than those of the acceptor-type defect.

calculation, ${ }^{7}$ a spacing of four air-hole rows was chosen for L3. In the case of L4, we found that the maximization of the drop efficiency requires more complex methods, which are described later.

The devices were fabricated by first drawing design patterns on the resist mask coating of a silicon-on-insulator substrate by electron-beam lithography. Resist patterns were then transferred to the Si layer using the inductively coupled plasma etching technique. The $\mathrm{SiO}_{2}$ layer under the patterned Si layer was selectively etched off to form the airbridge structure. Figures 1(b) to 1(e) show scanning electron microscope images of the fabricated devices from which geometrical distortions in the structures can be seen to be very small. Deviations between the fabricated and designed structures were of the order of a few nanometers.

First, a channel-drop experiment was carried out on the L3 defect device in which photons were injected from the waveguide facet and the radiation emitted vertically from the point defect was observed. The channel-drop spectrum is plotted in Fig. 2(b). As can be seen in Fig. 2(b), the full width at half maximum (FWHM) of the resonant peak is as narrow as $0.6 \mathrm{~nm}$, corresponding to a $Q$ value of about 2600, very close to the theoretical prediction (2900) described in a previous work. ${ }^{7}$ For comparison, the channel-drop spectrum of an acceptor-type defect-based device previously reported ${ }^{1}$ is also plotted in Fig. 2(a). In this case, the $Q$ value and FWHM are around 400 and $4.1 \mathrm{~nm}$, respectively. From Figs. 2(a) and 2(b), it is clear that the $Q$ factor of the L3 defect is six to seven times greater than that of the acceptor-type defect. These experimental results indicate that drastic improvements in the $Q$ factor of the filtering device have been Downloaded 06 Mar 2008 to 130.54.110.22. Redistribution subject achieved as theoretically predicted in our last paper. Here, we should note that the measured $Q$ factor of the device is not the intrinsic $Q$ factor of the cavity itself. The intrinsic $Q$ factor is determined by the coupling loss to the free space mode only. On the other hand, the measured $Q$ factor is also affected by the coupling loss to the waveguide mode. Therefore, the intrinsic $Q$ factor should be larger than the measured $Q$, which we actually confirmed on an experimental basis. Further details will be presented in a separate publication due to limitations in space.

In the expectation of further improvements in $Q$ factor, devices containing L4 defects were first theoretically investigated using a three-dimensional finite-difference timedomain method. ${ }^{10,11}$ The $Q$ factor of the L4 defect was found to increase by a factor of 3 over the L3 defect, with a slightly lower fundamental mode resonant frequency. At this lower frequency, the group velocity in the line-defect waveguide (a filled row of air holes) is too low to be applied to practical devices. The width of the waveguide was therefore increased a little $(0.11 a)$ to adjust the high group velocity region ${ }^{12}$ to match the fundamental mode of the L4 defect. In addition, the distance between the point and line defects $(d)$ was readjusted, since the coupling between the defects depends on changes in the lateral mode distribution of the line-defect waveguide. Drop efficiency was found to become maximal (48\%) for a separation $d$ of three rows, where the total $Q$ factor was calculated to be around 10000. Based on this theoretical analysis, the device was fabricated and dropping spectrum measured, as shown in Fig. 2(c). As can be seen, the $Q$ factor of the dropping peak is around 6400. This total $Q$ factor is approximately 16 times greater than acceptortype defect devices, and about 2.5 times higher than L3 defect devices. The difference between theoretical and experimental values is thought to be due to fluctuations in the fabricated structures and/or insufficient convergence of the calculation.

In addition to the high- $Q$ factor, the fact that the cavity has a single-resonant mode in the spectral range of concern is important for the application to the channel add/drop filters. From both calculation ${ }^{7}$ and experiment, both L3 and L4 defects are found to have only one resonant mode in a wide spectral range of more than $60 \mathrm{~nm}$, as shown in Figs. 2(b) and $2(\mathrm{c})$. These single-mode ranges are wide enough for the usual WDM applications.

Radiation patterns of the devices were also measured. Near-field images of the light emitted from the L3 and L4 defect devices are shown in Figs. 3(a) and 3(b), respectively. As can be seen in Figs. 3(a) and 3(b), each defect exhibits a different radiation pattern, with the L3 defect exhibiting a single spot while L4 exhibits two spots separated along the axis of the defect. For coupling to external optics, the efficiency of L4 is much lower than that of L3 due to the splitting of the radiation pattern. To verify the experimental results, radiation patterns of the defects were calculated using a previously reported method. ${ }^{7}$ Theoretical radiation patterns for the L3 and L4 defects are shown in Figs. 3(c) and 3(d), respectively, with white circles indicating the numerical aperture (NA) of the objective lens used in the measurements $(\mathrm{NA}=0.40)$. Experimental and theoretical results are in good agreement when the effects of the limited NA is conAlP license or copyright; see http://apl.aip.org/apl/copyright.jsp 


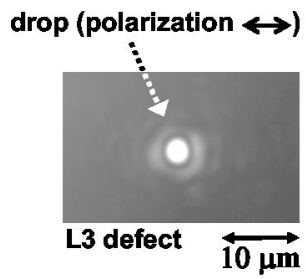

(a)

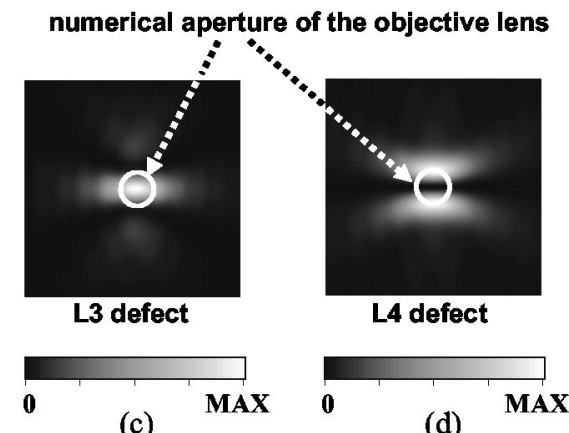

(c)

(d)

FIG. 3. (a) and (b) Measured microscopic images of radiation from devices consisting of L3 and L4 defects. The longitudinal direction of the letter coincides with the longitudinal axes of the defect and waveguide. NA of the objective lens used is 0.40. (c) and (d) Calculated radiation patterns from devices consisting of L3 and L4 defects. White circles indicate the range of experimentally observable angles as determined by the NA of the experimental setup.

sidered. Polarizations of the dropped light were also measured, with the results shown in insets in Figs. 3(a) and 3(b). In both cases, the light was found to be linearly polarized with the electric field perpendicular to the axis of the defects, also in agreement with theoretical results. ${ }^{7}$ Linear polarization of the dropped light is thought to be useful in most practical applications.

The reason for difference in the light-emission patterns of the two devices is investigated by examining the electricfield patterns of the fundamental defect modes of the defects. Figures 4(a) and 4(b) show the $x$ and $y$ components of the fundamental defect mode electric field across the center plane of the L4 defect device, and Figs. 4(c) and 4(d) show those of the L3 defect device that were presented in a previous work. ${ }^{7}$ For radiation perpendicular to the slab, radiation power is proportional to the DC component of the spatial Fourier transform of the defect mode electric field, which can
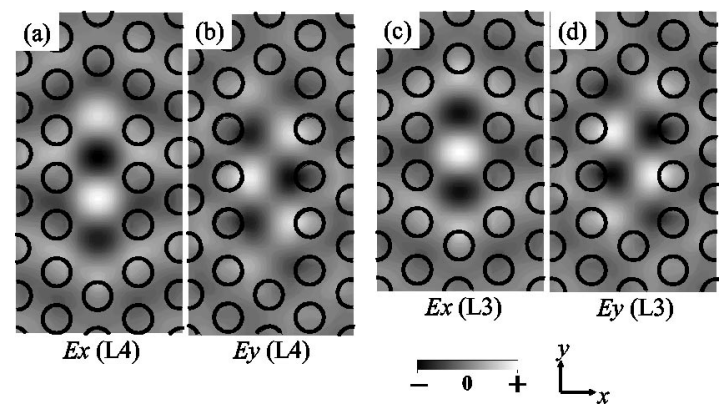

FIG. 4. Distributions of the $x$ and $y$ components of defect mode electric field for the (a) and (b) L4 defect, and (c) and (d) L3 defect (see Ref. 7). Only the $x$ component of the L3 defect mode electric field is even about both $x$ and $y$ axes, meaning that the spatial integration of electric field can be nonzero in this case. For the other cases, electric fields are odd about the $x$ and/or $y$ axes, and so the spatial integration of the electric field is zero. be calculated from a simple spatial integration of the electric field. Since $E_{x}\left(E_{y}\right)$ of the L4 defect mode are odd about the $x(y)$ axis as shown in Fig. 4(a) [Fig. 4(b)], the spatial integration of $E_{x}\left(E_{y}\right)$ inevitably becomes zero. Therefore, radiation perpendicular to the slab plane is prohibited for both polarizations, and the radiation pattern splits into two spots. On the other hand, $E_{x}$ of the L3 defect mode is even about both $y$ and $x$ axes as shown in Fig. 4(c), which typically yields a nonzero integration. Spatial integration of $E_{y}$ of the L3 defect mode yields zero since it is odd about both $y$ and $x$ axes. Therefore, only the light with $E_{x}$ polarization can be emitted vertically from the L3 defect. This leads to the general rule that the number of air holes filled should be odd to produce vertical radiation from the fundamental modes of line-shaped donor-type defects.

Finally, a brief description of the reasons for the $Q$ factor of the L4 defect being higher than that of the L3 defect is given. One reason is the difference in cavity sizes. Another reason is the deference in the symmetry of the in-plane mode fields ${ }^{13}$ with vertical emissions cancelled out in the case of the L4 defect. More importantly, a sharp decay in the defect mode field at the boundary between the defect and surrounding crystal was found to have a very large effect on $Q$ factors. Further details are to be presented in a separate publication due to limitations in space.

In summary, we have experimentally demonstrated that donor-type point defects are very useful for improving the filtering resolution of channel add/drop devices. The devices, which consist of line-shaped defects of three or four missing air holes, were shown to have very high filtering resolutions with $Q$ factors as high as 2600 and 6400, respectively. Light emitted from both of these defects is linearly polarized with the electric field perpendicular to the axes of the defects. The radiation pattern of the $\mathrm{L} 3$ defect is a single spot while that of the L4 defect is divided into two spots. Although simultaneous improvement of $Q$ factor and radiation patterns is a future task, these results are encouraging for the application of 2D PC slab devices to D-WDM communication systems.

This work is partly supported by Grant-in-Aid No. 14205012 from the Ministry of Education, Culture, Sports, Science, and Technology of Japan and by the International Communications Foundation.

${ }^{1}$ S. Noda, A. Chutinan, and M. Imada, Nature (London) 407, 608 (2000).

${ }^{2}$ A. Chutinan and S. Noda, Phys. Rev. B 62, 4488 (2000).

${ }^{3}$ A. Chutinan, M. Mochizuki, M. Imada, and S. Noda, Appl. Phys. Lett. 79, 2690 (2001).

${ }^{4}$ M. Imada, S. Noda, A. Chutinan, M. Mochizuki, and T. Tanaka, J. Lightwave Technol. 20, 873 (2002).

${ }^{5}$ O. J. Painter, J. Vučković, and A. Scherer, J. Opt. Soc. Am. B 16, 275 (1999).

${ }^{6}$ S. G. Johnson, S. Fan, P. R. Villeneuve, and J. D. Joannopoulos, Phys. Rev. B 60, 5751 (1999).

${ }^{7}$ Y. Akahane, M. Mochizuki, T. Asano, Y. Tanaka, and S. Noda, Appl. Phys. Lett. 82, 1341 (2003).

${ }^{8}$ J. Vučković, M. Lončar, H. Mabuchi, and A. Scherer, Phys. Rev. E 65, 016608 (2001).

${ }^{9}$ H. Y. Ryu, S. H. Kim, H. G. Park, J. K. Hwang, Y. H. Lee, and J. S. Kim, Appl. Phys. Lett. 80, 3883 (2002).

${ }^{10}$ K. S. Yee, IEEE Trans. Antennas Propag. 14, 302 (1966).

${ }^{11}$ G. Mur, IEEE Trans. Electromagn. Compat. 23, 377 (1981).

${ }^{12}$ M. Notomi, K. Yamada, A. Shinya, J. Takahashi, C. Takahashi, and I. Yokohama, Phys. Rev. Lett. 87, 253902 (2001).

${ }^{13}$ K. Srinivasan and O. Painter, Opt. Express 10, 670 (2002). 